Veritas Et Scientia

Vol. 7, N ${ }^{\circ} 2,1002-1010$

Julio - Diciembre del 2018.

ISSN Edición Online: 2617-0639

https://doi.org/10.47796/ves.v7i2.78

\title{
¿LOS USUARIOS INDUSTRIALES DE LA ZONA FRANCA DE RIONEGRO, HUMANIZAN LA LOGISTICA?
}

\author{
¿THE INDUSTRIAL USERS OF THE RIONEGRO'S FREE ZONE HUMANICE THE LOGISTICS?
}

\author{
MG. Claudia Elena Lukau Quintero ${ }^{1}$ \\ MG. Silvia Liliana Ceballos Ramírez ${ }^{2}$ \\ CPC. Gabriela Flores Mantegazza ${ }^{3}$ \\ Presentado: 25/11/2018 \\ Aceptado: 03/12/2018 \\ Publicado online:27/12/2018
}

\section{RESUMEN}

El comercio juega un papel importante en el desarrollo de un país o de una región y, los gobiernos para promover la demanda externa, la competitividad y la industrialización, comienzan a impulsar las zonas francas, que con la ayuda de la logística, permiten lograr la eficacia y eficiencia en los procesos que se desarrollan dentro de las empresas, las cuales en la gran mayoría de los casos, presentan una estructura jerárquica vertical y piramidal; es decir, los empleados no pueden tomar decisiones operativas en su día a día, pero cuando se implementan estrategias enfocadas al mejoramiento de la calidad de vida de los empleados se ve reflejado en una mejora en la productividad del personal. Por otra parte, la logística por su agilidad en las operaciones requiere de un líder que identifique las fortalezas del recurso humano y de ese modo potencializar el trabajo en equipo. Surge entonces la pregunta $¿$ si los usuarios industriales de la zona franca de Rionegro, humanizan la logística? El hallazgo principal fue que los Usuarios Industriales de esta Zona Franca promueven el buen trato, generando armonía en los equipos de trabajo y, donde la persona es el recurso más importante para dichas organizaciones.

Palabras claves: Logística, Humanización, Usuario Industrial, Zona Franca.

\begin{abstract}
The trade plays a very important role in the development of the country or the region, the governments to promote de external demand, the competitiveness and industrialization, begins to impulse the free zone, with the help the logistics, allows to make the effectiveness and efficiency in the products that are developed inside the business, with most of them cases, the show a hierarchical structure, vertical and pyramidal; by that means, the employees can not

\footnotetext{
${ }^{1}$ Universidad Católica de Oriente. UCO Docente del Programa de Comercio Exterior - FACEA.

${ }^{2}$ Universidad Católica de Oriente. UCO Docente del Programa de Comercio Exterior - FACEA.

${ }^{3}$ Universidad Privada de Tacna - UPT. Docente de la Carrera Profesional de Administración de Negocios Internacionales.
} 
make operative decisions in day to day, but when implemented strategies focused in the improvement of the quality of life in the employees shows an improvement in the personal productivity. on the other hand, the logistic by heir agility in the operations requires a leader that identifies the strengths and human resources and thereby potentiate team work. so a question arises iif the industrial users of the Rionegro's free zone promotes good treatment, generating team harmony, where the person is the most important resource for the organization Keywords: Logistics, Humanization, Industrial User, Free Trade Zone.

"los clientes no son lo primero, lo primero son los empleados. Si cuidas a tus empleados, ellos cuidaran a tus clientes" Richard Branson.

\section{INTRODUCCION}

La logística, es una actividad interdisciplinaria que vincula diferentes áreas de la compañía, requiere de una dirección eficiente, que permita conocer las necesidades de los clientes y poder así, agregar valor, administrar los recursos que vinculan (Valdez y Hernández 2010) (1), su misión es coordinar las entregas exactas, en el tiempo correcto y con el menor costo posible (Mora, 2016) (2), se deben realizar investigaciones que mejoren los sistemas y generar procesos de diferenciación con respecto a la competencia (Bohórquez y Puello, 2013) (3). Los beneficios que presenta la logística para una empresa son: el incremento de la competitividad y la rentabilidad, optimizando la gerencia y gestión de los procesos, facilitado la coordinación de los factores que influyen en la decisión de compra y planifica las actividades internas y externas de la empresa. La logística es un aliado del sector empresarial, para que sea eficiente se debe conocer las actividades internas como el pronóstico de la demanda, la administración de inventarios, manipulación de materiales, procesamiento de órdenes, empacado, selección de planta y bodegas, aseguramiento del abastecimiento, logística inversa y almacenamiento. Igualmente hay actividades logísticas externas como el servicio al cliente, comunicación logística, servicio de soporte y transporte (Bohórquez y Puello, 2013) (3).

Cos y Navascués, (2001) (4), aducen que el principal objetivo de la logística está en priorizar las necesidades de los clientes, garantizando una ventaja competitiva a través del menor costo y flexibilizar la distribución para acoplarse fácilmente a la necesidad de un mercado en constante cambio. Rozo (2014) (5) presenta la importancia de enlazar los objetivos de la logística con los inventarios, almacenamiento, transporte, proceso $\mathrm{y}$, por último, el equipo de trabajo que tiene como función fundamental un óptimo manejo del aprovisionamiento, las compras el almacenamiento y la gestión del stock.

Por otro lado el concepto de cadena de suministro o canal logístico, subraya el encadenamiento de las operaciones logísticas de las partes que se ven involucrados de manera directa o indirectamente al cliente e incluye a fabricantes, proveedores, transportistas, almacenistas y vendedores (Chopra y Meindl, 2008) (6), eliminando actividades redundantes que no agregan valor; si esta, está bien estructurada puede ayudar al éxito de la empresa ya que garantiza la trazabilidad en cada una de las operaciones, permitiendo la mejora continua.

Según la Real Academia de la Lengua -RAE- (2017) (7) Humanizar es hacer humano, familiar $\mathrm{y}$ afable a alguien o algo. El concepto epistemológico de Humanización se encuentra en el centro de un amplio debate. (Ayres, 2005; Casate y Correa, 2005; Casimiro, 2012; Ceccim y Merhy, 2009, Chernicharo, Freitas y Ferreria, 2013; Deslandes, 2004; Isayama et al., 2011; Oliveira y kruse, 2006; Santos Filho, Barros y Gomez, 2009; Tavares y Souza, 2008; Waldow y Borges, 2011). Es un concepto polisémico con múltiples acepciones. (Beck et al., 2007; Maciak, 2008; Risotto, 2002; Simoes, Rodrigues, Tavares y Rodrigues, 2007; Souza y Moreira, 2008). Afirman que la humanización es una expresión de difícil conceptualización, teniendo en cuenta su carácter subjetivo, complejo y multidimensional (cita de Valenzuela, 2015) (8). Ceballos (2012) (9) describe la humanización como el abordaje íntegro del ser humano, que interrelaciona dimensiones biológicas, conductuales y psicológicas; en una exploración del bienestar propio y de los demás ante cualquier circunstancia. Humanización es el acto de compenetrarse y entender que los seres humanos ocupan un sitio exclusivo en este universo y a 
su vez las únicas criaturas capaces de entenderlo. Esta, se ha ido abordando desde las ciencias de la salud, donde el servicio al paciente requiere una asistencia integral con calidez y que responda a todas las dimensiones humanas (Correa, 2016) (10).

"La humanización hace referencia al abordaje integral del ser humano, donde interactúan las dimensiones biológica, psicológica y conductual, mediante la búsqueda del bienestar propio y de los demás ante cualquier circunstancia" (Hoyos, Cardona y Correa, 2008) (11). La logística no puede ser ajena, ya que la gerencia logística moderna debe coexistir con un liderazgo en los diferentes ámbitos de la organización, con directores que, más que un conocimiento técnico, tengan habilidades de influir en los demás de manera positiva para alcanzar los objetivos propuestos. Un líder logístico no mide las horas concentradas en el trabajo sino la capacidad de alcanzar el mejor resultado con el menor desgaste del equipo, en palabras de Adela Cortina las empresas que conducen éticamente, son la que terminan siendo competitivas. El factor humano es un elemento clave en la estrategia corporativa, un empleado satisfecho desarrolla con más entusiasmo su labor y se traslada en beneficios para la compañía.

El hombre posee necesidades insatisfechas y gracias a ello se da el comercio, ante la necesidad de movilizar los bienes se han desarrollado procesos logísticos, los gobiernos comienzan a establecer mecanismos de facilitación del comercio, entre dichas figuras se encuentran las zonas francas, cuyo objetivo es convertirse en polo de desarrollo en la región donde se ubica, promoviendo la competitividad $\mathrm{y}$, al ser destinados prioritariamente para el mercado internacional y de forma subsidiaria al mercado nacional, donde se simplifican procedimientos que permiten las entregas exactas, en el menor tiempo y costo posible.

Se denomina Zona Franca, al área geográfica delimitada dentro del Territorio Aduanero Nacional - TAN- en donde se desarrollan actividades industriales de bienes y de servicios, o actividades comerciales, bajo una normatividad especial en materia tributaria, aduanera y de comercio exterior (Echavarría, Gaviria y Muñoz, 2017) (12). Con la Ley 105 de 1958 (13), se crea la Zona Franca Industrial y Comercial de Barranquilla; en la década de los setenta entraron en funcionamiento las zonas francas de Buenaventura, Cartagena, Cúcuta, Palma seca y Santa Marta. Las zonas francas en Colombia han sufrido cambios normativos importantes desde 1985, los cuales eran establecimientos públicos y bajo la ley 7 de 1991 (14) se convierten las ya creadas en privatizadas y las nuevas en privadas. En 1993, nace la primera zona Franca Permanente de carácter privado y se denomina Zona Franca de Rionegro, la cual, se encuentra ubicada a 30 kilómetros de Medellín, cerca al aeropuerto internacional José María Córdova, en ella están instaladas 97 empresas calificadas como usuarios Industriales de Bienes, Servicios y Comerciales; tiene un área aproximada de $150.000 \mathrm{~m} 2$, es considera una de las más productivas del departamento de Antioquia, la cual genera más de 3000 empleos directos y mil indirectos (Promotora Nacional de Zonas Francas, 2018) (15).

Según el Departamento Nacional de Estadísticas -DANE- (2017) (16), de las tres zonas francas que operan en Antioquia, la de Rionegro reportó ventas al extranjero por 94,7 millones de dólares, lo que representa un incremento del 13 por ciento en comparación con 2016.

La importancia de esta investigación radica en la estrecha relación que existe entre la zona franca, la logística y el recurso humano que interactúa en las operaciones de comercio exterior. El 90\% de los empleos que se generan en una zona franca son para personas que no tienen un nivel de formación avanzada; y la rapidez de las operaciones logísticas pueden hacer que la atención se centre en los procesos y se dejen olvidadas a las personas. El objetivo era conocer si los usuarios industriales de la zona franca de Rionegro, humanizan la logística.

\section{METODOLOGÍA}

El tipo de investigación, inicialmente fue exploratorio para hacer un acercamiento al panorama de la humanización en la Zona Franca de Rionegro. Luego se pasó, a una investigación descriptiva para contrastar con el grupo de personas entrevistadas y encuestadas, si existían estrategias de humanización en las empresas que se tomaron como muestra; en este proceso se examinaron las variables a trabajar, selección de la técnica para recolectar datos y fuentes a 
utilizar. El método de investigación fue un enfoque mixto, es decir cuantitativo y cualitativo. La investigación cualitativa, permitió recoger información mediante la interacción con los gerentes, dueños y jefes de personal de algunas empresas de la Zona Franca. Con la investigación cuantitativa la información recogida, fue tabulada, llevada a datos numéricos con la ayuda de la estadística, para poder hacer generalizaciones. La Unidad de análisis fueron los usuarios Industriales de la Zona Franca de Rionegro. El Universo con que se contaba en el 2017 era de 65 empresas, para determinar la muestra, se utilizó la fórmula de poblaciones finitas, con un nivel de confianza del $95 \%$, un intervalo de confianza de $2.15 \%$, dando como resultado 49 encuestas. Los instrumentos utilizados fueron la entrevista semiestructurada y una encuesta. La entrevista semiestructurada se aplicó a dueños, gerentes y jefes de recurso humano de 14 empresas reconocidas como usuarios industriales ubicados en la zona Franca de Rionegro. El formato cuenta con una identificación de la empresa y la persona que se entrevistó. Se tenían planteadas ocho (8) preguntas abiertas; como era una conversación, estas preguntas se podían cambiar, modificar o ampliar de acuerdo a la dinámica que se daba. Se presentó el consentimiento informado, para que las personas autorizaran su participación y grabación; para su análisis se utilizó el Software Atlas. Ti. La encuesta, tenía un formato predeterminado con veinte seis (26) preguntas abiertas, cerradas y dicotómicas, la cual se aplicó a 49 trabajadores de diferentes empresas reconocidas como Usuarios Industriales de la Zona Franca y su tabulación se realizó en Excel. La técnica utilizada fue estadísticas para cuantificarlas y técnicas cualitativas para interpretarlas.

\section{RESULTADOS}

Una mirada de la humanización de la logística por parte de la alta gerencia de los usuarios industriales de la ZF de Rionegro

De acuerdo a la base de datos suministrada por la Zona Franca de Rionegro (2017), se encontró que hay 65 empresas reconocidas como Usuarios Industriales, de los cuales se entrevistaron 14 personas entre los que se encontraban los dueños, gerentes, jefes de recurso humano, de bodega y logística. Frente a la pregunta que es lo más importante para las empresas, 5 de ellas $(36 \%)$ consideran que las personas son esenciales para el logro de los objetivos trazados, para 4 $(28.5 \%)$ es la empresa el eje esencial, para $2(14,3 \%)$ son primero los procesos y, para 3 $(21.2 \%)$ es necesario la conjugación de los empleados, procesos y empresa para un desempeño eficiente.

Las empresas hacen declaraciones desde la misión, visión y los valores en ellas se definen y representan el por qué y para qué existen, se comprometen con sus empleados, clientes, socios y el entorno. De las 14 empresas, solo 2 entrevistadas (14\%), en su página web presentan estos tres elementos y solo una de ellas declara la importancia de que sus empleados estén en mejores condiciones y la otra presta importancia a la calidad de los productos y la rentabilidad; para 11 empresas que representan $78.58 \%$ reconocen lo importante de capacitar, empoderar y desarrollar procesos de liderazgo para los empleados. Los temas que más se trabajan desde gestión del talento humano son para el desarrollo de las habilidades técnicas necesarias para la realización del trabajo en el día a día y son del régimen franco, manejo de operaciones, régimen aduanero y cambiario, logística, almacenamiento, material handling, sistema de administración de riesgos lavados de activos y financiación de terrorismo - SARLAFT -, Business Alliance for Secure Commerce -BASC- y de calidad. Para el desarrollo de habilidades blandas se trabajan temas de liderazgo, comunicación, trabajo en equipo, procesos de convivencia, seguridad y salud en el trabajo. En un mundo donde la competencia es una constante, las empresas deben desarrollar estructuras más fuertes y asegurar la calidad en los procesos, evitar los desperfectos para asegurar la productividad, pero es normal que en las empresas los trabajadores cometan errores y para ello hay un debido proceso. El coordinador de cada proceso con el empleado analiza la situación e identifican el error y se retroalimenta, se capacita nuevamente para que no pase, se desarrollan planes de mejora, se sanciona de acuerdo al reglamento de trabajo con los 
llamados de atención y anotaciones en la hoja de vida, estas alertas se hacen de acuerdo al nivel de gravedad del error cometido por el empleado.

Para Yepes (2017) (17), la humanización en las empresas, se trata de entender de que cada miembro de una organización se levanta todos los días no a trabajar, sino a vivir. Entonces, ¿por qué no hacer que esa vida sea buena para todos? Sin duda, parece que estamos ante una fuerza centrífuga que estuviera alejando lo humano de las cosas más sencillas de la vida. Leí una frase que me marcó: 'muchas veces lo que menos hacemos los humanos es ser humanos'. Se nos olvida que somos seres colectivos y fruto de las relaciones. Así que es muy fácil hablar de coherencia, inclusión, cercanía, respeto, pero lo difícil es darles contenido a esas palabras.

Las definiciones de humanización que se encontraron, en el proceso de entrevista dada por los empleadores son las siguientes:

- Dar valor a cada integrante de la organización, ver a los trabajadores como seres humanos que necesitan apoyo en su vida diaria.

- Respeto por las personas, no generar desigualdades, confianza y apoyo

- Es un proceso en el cual, las empresas buscan cultivar hábitos saludables desde el ambiente laboral y personal para que fluyan las relaciones laborales, es decir, son más sensibles con el empleado.

- Generar espacios de reconocimiento, beneficios, trabajos con actividades que no se exceden de los tiempos determinados para la labor, flexibilización de horarios. Propiciar espacios de diálogo. Salarios dignos y acordes con la labor y formación. Tiempo para la familia.

Contrastando la cita de Yepes y las respuestas dadas por los empleadores se puede decir que existe una alta correlación de conceptos. La definición es clara, no obstante, llevarlo a la práctica es el reto para todas ellas. Los 14 empleadores, consideran que no solo la logística se puede humanizar, todas las actividades y procesos son susceptible a ello. Sólo depende de que la alta dirección tome la decisión de llevarlo a la realidad.

De los entrevistados 6 empresas no realizan ninguna actividad para humanizar la logística; las 8 restantes consideran que jornadas de trabajo justas, flexibilización de horarios, fomentar la creatividad y el aprendizaje, el respeto entre todos los miembros de la organización, jornadas de capacitación desde el ser, motivación e incentivos para realizar buenas practicas logísticas, generar un clima de confianza, desarrollar programas de comunicación asertiva, ser sensibles y no permisivos, empoderamiento al personal, información compartida y relevante en toda la pirámide, desarrollo de programas de formación de líderes para que den un buen trato y creación consolidación del departamento de logística, planear y ejecutar las actividades del día a día, e implementar gerente por un día en las empresas; son formas que permiten empezar a dar pasos para humanizar este proceso tan importante dentro de todas las organizaciones.

\section{Una mirada de la humanización de la logística por parte de los empleados de los usuarios industriales de la ZF de Rionegro}

En cuanto a la relación que tienen como empleados con el jefe inmediato, un $88 \%$ de ellos afirman, que el superior a cargo propone objetivos prudentes, de acuerdo a la realidad de la empresa y a las exigencias del cliente; sienten que el jefe confía en las capacidades del equipo de trabajo, saben acerca de los procesos, cuentan con el jefe como un apoyo para dar soluciones y hacer que todo sea posible; el 10\%, manifiesta que las metas son imposibles de alcanzar, el jefe no está centrado en las labores del equipo y no conoce bien el desarrollo de la operación y un $2 \%$ no responde. Además, el superior a cargo es respetuoso con ellos, habla en buenos términos, da respuestas amables y es de buenos modales. El $90 \%$ de ellos, considera que la forma de ser del jefe es un buen ejemplo a seguir, se ha ganado la admiración de los empleados, es una persona con experiencia, ha sacado la empresa adelante y se preocupa por tener todo a tiempo; el 10\% expresa que se enoja con facilidad e indispone al equipo de trabajo, no conoce los procesos, en ocasiones no se expresan bien y son patronistas. El 92\% de los encuestados, 
consideran que los llamados de atención realizados por el superior se hacen de manera adecuados, ya que dan consejos y recomendaciones a seguir, actúan con prudencia y lo hacen en buenos términos. El 8\% ha recibido llamados de atención en público y regaños sin argumentos. El 96\% de los encuestados, afirman que cuando tienen problemas personales el jefe es accesible, si necesitan un permiso está dispuesto a darlo, es una persona humana y comprensible con los problemas, escucha y ayuda a dar soluciones. El otro $4 \%$, dicen que los problemas personales no deben incidir en el ámbito laboral. Los factores que, para los encuestados, puede afectar el clima laboral son: el trabajo bajo presión, las relaciones interpersonales, la falta de apoyo, los malos comentarios, e mal manejo de los conflictos grupales, la falta de comunicación, el incumplimiento con el pago, el mal trato, la envidia, la carga laboral, la falta de comunicación entre los departamentos y la preferencia por algunos empleados.

E1 73\% de los encuestados expresan que la empresa no tiene programas de humanización y el $27 \%$ dicen que la empresa brinda capacitaciones enfocadas hacia su bienestar.

Las recomendaciones que hacen los empleados, para que las empresas donde laboran humanicen la logística son: capacitaciones permanentes sobre los procesos logísticos, humanización y liderazgo, comprensión, acompañamiento y participación del jefe en todos procesos y enfocarse más en el ser que en el hacer.

Los encuestados opinan que los cambios tecnológicos que se desarrollan en la logística son buenos, siempre y cuando se incorpore el buen trato al ser humano, ya que ayudan a ser más ágiles. El conocimiento de nuevas herramientas abre puertas a la productividad, disminuyen costos y tiempos y, son necesarios para cumplir con los procesos. La tecnología ayuda a humanizar la logística, pues facilita la operación diaria, son herramientas ágiles, dinamiza los procesos, la automatización minimiza el desgaste del ser humano generando tiempo libre que puede ser dedicado a otras actividades de la empresa. Sin embargo, las empresas dotadas de tecnologías de punta han olvidado al ser como eje central de toda organización.

Haciendo un cruce de algunas variables con las respuestas dadas por empleadores y empleados, se encuentran descrita en la tabla 1, mostrando coherencia entre las partes, denotándose que los empleados consideran que las empresas donde trabajan los tratan como personas.

Tabla 1. Cruce de variables

\begin{tabular}{|c|c|}
\hline Respuesta de empleadores & \\
\hline $\begin{array}{l}\text { Cuándo hay errores en el equipo de } \\
\text { trabajo, ¿Cómo es el proceso de } \\
\text { llamado de atención? }\end{array}$ & $\begin{array}{l}\text { Cuando el superior le llama la atención } \\
\text { a alguien, ¿lo hace adecuadamente? }\end{array}$ \\
\hline $\begin{array}{l}\text { El coordinador de cada proceso con el } \\
\text { empleado analiza la situación e } \\
\text { identifican el error y se retroalimenta, } \\
\text { se capacita nuevamente para que no } \\
\text { pase, se desarrollan planes de mejora, } \\
\text { se sanciona de acuerdo al reglamento } \\
\text { de trabajo con los llamados de } \\
\text { atención y anotaciones en la hoja de } \\
\text { vida, estas alertas se hacen de acuerdo } \\
\text { al nivel de gravedad del error cometido }\end{array}$ & $\begin{array}{l}\text { El } 92 \% \text { de los encuestados, consideran } \\
\text { que los llamados de atención } \\
\text { realizados por el superior se hacen de } \\
\text { manera adecuados, ya que dan } \\
\text { consejos y recomendaciones a seguir, } \\
\text { actúan con prudencia y lo hacen en } \\
\text { buenos términos. El } 8 \% \text { ha recibido } \\
\text { llamados de atención en público y } \\
\text { regaños sin argumentos. }\end{array}$ \\
\hline $\begin{array}{l}\text { ¿Qué programas desarrolla la empresa } \\
\text { en temas de capacitación, } \\
\text { empoderamiento, liderazgo, entre otras } \\
\text { para los empleados de la empresa? } \\
\text { Para el desarrollo de las habilidades }\end{array}$ & $\begin{array}{l}\text { ¿La empresa le ofrece cursos de } \\
\text { capacitación? } \\
\text { El } 67 \% \text { de los empleados, expresa que } \\
\text { la organización ofrece cursos de } \\
\text { capacitación en temas de comercio }\end{array}$ \\
\hline
\end{tabular}


técnicas necesarias para la realización de su trabajo en el día a día, se hacen capacitaciones de: régimen franco, manejo de operaciones, régimen aduanero y cambiario, logística, almacenamiento, material handling, sistema de administración de riesgos lavados de activos y financiación de terrorismo - SARLAFT -, Business Alliance for Secure Commerce BASC-, calidad.

Para el desarrollo de habilidades blandas se trabajan temas como: liderazgo, comunicación asertiva, trabajo en equipo, procesos de convivencia, seguridad y salud en el trabajo.

¿Qué es para usted humanización?

Dar valor a cada integrante de la organización, ver a los trabajadores como seres humanos que necesitan apoyo en su vida diaria.

Respeto por las personas, no generar desigualdades, confianza y apoyo.

Es un proceso en el cual, las empresas buscan cultivar hábitos saludables desde el ambiente laboral y personal para que fluyan las relaciones laborales, es decir, son más sensibles con el empleado.

Generar espacios de reconocimiento, beneficios, trabajos con actividades que no se exceden de los tiempos determinados para la labor, flexibilización de horarios. Propiciar espacios de diálogo. Salarios dignos y acordes con la labor y formación.

Es pensar más en el ser que en el hacer. exterior, actualización en el régimen franco y logística para el desarrollo de las habilidades técnicas, lo que ayuda a aclarar temas relacionados con la operación, aumentan el conocimiento. Sin embargo, a los empleados les gustaría recibir capacitación en inglés, seguridad informática, manejo de personal y primeros auxilios, lo que ayudaría con la seguridad del trabajador y el trabajo en equipo.

Con respecto a las habilidades blandas necesarias para el desarrollo del trabajo, cuenta con capacitaciones en temas como: alimentación saludable, prevención del alcoholismo, comunicación asertiva, liderazgo y trabajo en equipo.

El 33\% no recibe ningún tipo de capacitación por parte de la empresa. Para el $80 \%$ de los trabajadores, cuando se les da cursos de capacitación es una forma de que la empresa muestre interés por su desarrollo personal y motivarlos. El $20 \%$ no cuenta con estos espacios.

Qué entiende usted por humanización? Es la manera como se mira más el aspecto de las personas que aportan a la empresa y no como máquinas de trabajo.

Comprender el lado natural, personal y sentimental de un individuo.

Preocuparse por el bienestar del ser humano, saberlas tratar en el entorno.

Trata que el trabajador se desgaste menos y rinda al 100\%.

Es saber que todos valemos mucho.

Es un ambiente de hermandad entre jefes y empleados.

Es el trato correcto y asertivos con las personas

Buen trato y acorde con las capacidades del empleado.Es que dentro de los procesos se tenga cuidado con el desarrollo integral del individuo.

Fuente: elaboración propia, a partir de la información recopilada, 2018

\section{CONCLUSIONES}


Actualmente, la palabra estrategia es utilizada en diferentes contextos, convirtiéndose en un concepto de uso generalizado. Para Chandler (2003) (18), la estrategia es la determinación de las metas y objetivos de una empresa a largo plazo, las acciones a emprender y la asignación de recursos necesarios para el logro de dichas metas. Aunque, las empresas encuestadas aducen no tener un plan estructurado de humanización de los procesos logísticos que desarrollan, sin embargo, realizan un sinnúmero de actividades, que hacen que los empleados se sientan respetados, valorados, escuchados y reconocidos. Se sienten importantes en la organización, consideran que no hay desigualdades, existe confianza en el equipo de trabajo, generando un buen ambiente laboral. Las empresas tienen jornadas laborales justas, generando estímulos de diferente índole, para que las personas cumplan con las metas propuestas por la compañía; mostrando, que un empleado satisfecho es el reflejo en el progreso, eficiencia y competitividad de la empresa. Humanizar no es solo diseñar planes, metas, estrategias que favorezcan únicamente a los intereses de las empresas; es manejar adecuadamente los valores, las actitudes, promover la dignidad, el empoderamiento del otro, acompañarlo y consolidarse como grupo. Pero para que se de esto, debe existir coherencia entre el planteamiento de los programas que realiza la empresa y su puesta en marcha para mejorar la calidad de vida de las personas. Un equipo de trabajo humanizado, presenta autonomía, armonía, colaboración, respeto, tolerancia, actitudes y habilidades que facilitan su labor, permitiendo crecer como personas y profesionales. Las empresas entrevistadas en la zona franca de Rionegro, generan una experiencia grata para los trabajadores, permitiendo concluir que, las operaciones logísticas se encuentran humanizadas, que no son tan mecanizadas como se pensaría y son sustentadas por la actitud de las personas, el liderazgo de los superiores y el respeto por el otro. La solidaridad es necesaria para el desarrollo del liderazgo empresarial, la educación, la ética en la toma de decisiones económicas y empresariales sean el elemento diferenciador.

Finalmente, en la zona franca los usuarios industriales sí humanizan la logística. Es una tarea que les compete a todos, entender que se es parte de una organización, que se trabaja, que se desea generar utilidades, pero más importante aún que se trabaja con seres llamados a vivir conscientes del entorno que les rodea, ser empáticos poniéndose en el lugar del otro, ser integro, crear cultura empresarial, apostarle a la confianza a la creatividad y a la motivación. Para ser jefe hay que ser primero buena persona, para transformar una compañía primero hay que transformarse a sí mismo a través del auto conocimiento; es una lucha constante donde vivir en armonía sea la meta y cuando logremos entender que es el ser humano, lograremos vivir en paz. Actualmente, las personas se ven enfrentadas a un sinnúmero de presiones, carreras, obligaciones, pero a algunos empleadores se les olvida que trabajan con seres humanos, que necesitan comprensión, que tienen debilidades y fortalezas. Algunos pasos básicos para humanizar las operaciones son: conocer mejor a los empleados, realizar actividades que fortalezcan el entorno familiar, ya que esto beneficia el aspecto laboral, elaborar protocolos internos que permitan detectar y dar solución a conflictos internos, ofrecer condiciones laborales adecuadas, que contribuyan a equilibrar el salario monetario con el emocional, hacer procesos de aproximación a la cultura de la empresa, establecer mecanismos de comunicación interna eficientes, gestionar las expectativas del trabajador, generando un ambiente de confianza, sinceridad y respeto y recordar que ante todo trabaja con personas, que se puede humanizar, sin olvidar la productividad.

\section{REFERENCIAS BIBIOGRÁFICAS}

(3) Bohórquez y Puello. (2013). Diseño de un modelo de gestión logística para mejorar la eficiencia organizacional de la empresa coralinas \& pisos s.a. corpisos s.a. en el municipio de Turbaco, bolivar. Recuperado de: http://190.242.62.234:8080/jspui/bitstream/11227/468/1/TESIS.pdf

(9) Ceballos, C. (2012). Humanización de la Atención en Salud. Recuperado de: http://bdigital.ces.edu.co:8080/repositorio/bitstream/10946/1675/2/ Humanizacion_servicios_salud.pdf 
(13) Colombia. Congreso de Colombia. Ley 105 de 1958. (diciembre 31 de 1958). Por la cual se crea la Zona Franca de Barranquilla y se autoriza la creación de otras.

(14) Colombia. Congreso de Colombia. Ley 7 de 1991. (enero 16 de 1991). Por la cual se dictan normas generales a las cuales debe sujetarse el Gobierno Nacional para regular el comercio exterior del país, se crea el Ministerio de Comercio Exterior, se determina la composición y funciones del Consejo Superior de Comercio Exterior, se crean el Banco de Comercio Exterior y el Fondo de Modernización Económica, se confieren unas autorizaciones y se dictan otras disposiciones

(10) Correa, M. (2016) La Humanización de la atención en los servicios de salud: un asunto de cuidado.

Recuperado

de: https://www.revistacuidarte.org/index.php/cuidarte/article/view/300

(4) Cos y Navascués. (2001). Manual de logística Integral. Madrid: Ediciones Díaz de Santos S.A Recuperado de: https://books.google.es/books? $\mathrm{hl}=$ es\&lr=\&id=dxTImJ4ipCMC\&oi=fnd\&pg=PR21\&dq=logistica\&ots=5p6ITnaH6V \& sig $=$ azLsrCgFeNx3HX7jV-RjOO3Sn68\#v=onepage \& $\mathrm{q}=$ logistica\& $\mathrm{f}=$ false

(18) Chandler, A. D. (2003). Strategy and Structure. Chapters in the history of the American Industrial Enterprise. New York: Beard Books.

(6) Chopra y Meindl. (2008). Administración de la cadena de suministros. Recuperado de: https://alandap.files.wordpress.com/2015/10/administracion-de-la-cadena-desuministro-estrategia-planeacion-y-operacion-sunil-chopra-peter-meindl.pdf

(16) Departamento Nacional de Estadísticas - DANE- (2017). Boletín de Zonas Francas: Recuperado de:

https://www.dane.gov.co/files/investigaciones/boletines/Zonas_Francas/

Bol_Zonas_Francas_jun_2017.pdf

(12) Echevarría, Gaviria y Muñoz. (2017) Causas que generan la pérdida de participación de las exportaciones provenientes de la Zona Franca Permanente de Rionegro en el total de las exportaciones de Colombia. Recuperado de: http://www.bibliotecadigital.usb.edu.co/bitstream/10819/4295/1/Causas_Generan_Perdi da_Cortes_2017.pdf

(11) Hoyos, Cardona y Correa (2008). Humanizar los contextos de salud, cuestión de liderazgo. Recuperado de: file://C:/Users/clukau/OneDrive\%20-\%20UCO/Descargas/ DialnetHumanizarLosContextosDeSaludCuestionDeLiderazgo-3259045.pdf

(2) Mora, L. (2016). Gestión logística integral. Recuperado de: https://www.ecoeediciones.com/wp-content/uploads/2016/12/Gestion-logistica-integral2da-Edici\%C3\%B3n.pdf

(7) Real Academia de la Lengua, (RAE.). (2017) Recuperado de: http://dle.rae.es/? id=KnbmIsLghhhhhhhhhhhhhhhhhhhhhh

(5) Rozo, A. (2014). Gerencia Logística: Estrategias y Análisis en la Cadena Logística. Recuperado de: http://www.esumer.edu.co/images/centroeditorial/Libros/fei/libros/gerencialogistica.pdf

(8) Valenzuela, M. (2015). ¿Es posible humanizar los cuidados de enfermería en los servicios de urgencia? Recuperado de: https://rua.ua.es/dspace/bitstream/10045/50212/1/tesis_martina_valenzuela_anguita.pdf

(1) Valdez y Hernández. (2010). logística: evolución histórica y relevancia en el contexto actual cubano. Recuperado de: http://xn--caribea-9za.eumed.net/wp-content/uploads/logistica.pdf

(17) Yepes, C. (2017). Por otro Camino. Bogotá: Penguin Random House Grupo Editorial, S. A. S.

(15) Promotora Zona Franca Rionegro. (2018). Quienes Somos. Recuperado de: $\mathrm{http} / / / \mathrm{www} . z o n a f r a n c a r i o n e g r o . c o m / q u i e n e s . p h p$ 